\title{
Optical and thermal studies on pure and doped L-arginine phosphate crystals
}

\author{
P. Sumithraj Premkumar, X. Sahaya Shajan and H. Alex Devadoss
}

Centre for Scientific \& Applied Research, School of Basic Engg. \& Sciences, PSN College of Engg. \& Technol., Melathediyoor, Tirune/veli-627152, India. shajan89@gmail.com

\begin{abstract}
In the present study the single crystal growth of the pure and doped organic nonlinear optical crystal, L-arginine phosphate is reported. The dopants used were urea and thiourea. The crystals were grown by slow evaporation technique. The presence of various functional groups in the grown crystal was confirmed by Fourier transform infrared (FT-IR) analysis. The thermal properties of the grown crystals were studied by thermo gravimetric and differential scanning calorimetry analysis (TG/DSC). Second harmonic generation (SHG) measurement was used to confirm the organic nonlinear optical properties of the grown crystal.
\end{abstract}

Keywords: Growth from solutions; nonlinear optical materials; thermal analysis; doped LAP.

\section{Introduction}

Organic nonlinear optical (NLO) materials attract a great deal of attention due to their applications in optical devices, such as optical switches, optical modulators, optical communications, optical data storage etc. (Wong et al., 1996; Dmitriev et al., 1999; Gnanasekaran \& Madhavan, 2008; Munirathnam \& Madhavan, 2009). Among the organic class of compounds, $\alpha$-amino acids exhibit some specific features such as molecular chirality, weak Van der Waals and hydrogen bonding, the absence of strongly conjugated bonds, wide transparency ranges in the visible and UV spectral regions and zwitterionic nature which favours crystal hardness (Nicoud \& Twieg, 1987). L-arginine phosphate [LArg: $\mathrm{H}_{3} \mathrm{PO}_{4} \cdot \mathrm{H}_{2} \mathrm{O}$, L-Arg. is $\mathrm{NH}_{2} \mathrm{C}(\mathrm{NH}) \mathrm{NH}\left(\mathrm{CH}_{2}\right) 3 \mathrm{CH}\left(\mathrm{NH}_{2}\right)(\mathrm{COOH})$, ab -breviated as LAP] has been found to be a very promising NLO material (Xu et al., 1983). L-arginine phosphate (LAP) crystals can be grown from aqueous solutions. It is a biaxial crystal and belonging to the monoclinic system with space group $P 2{ }_{1}$. The lattice parameters are $a=10.75 \AA, b=7.91 \AA, c=7.32 \AA$ and $\beta=98^{\circ}$. Salient features of LAP that make it a better choice in NLO applications are wide transparency range, high optical damage threshold power, high non linear optical coefficient $(>1 \mathrm{pm} / \mathrm{V})$ and less hygroscopic nature than KDP crystals (Dhanraj et al., 1991; Ravi et al., 1994). In the present work, pure urea and thiourea doped LAP crystals were grown by the slow evaporation method as reported by the earlier authors. The

Research article

CIndian Society for Education and Environment (iSee)
Fig. 1. Photographs showing the grown pure and doped $L A P$ crystals

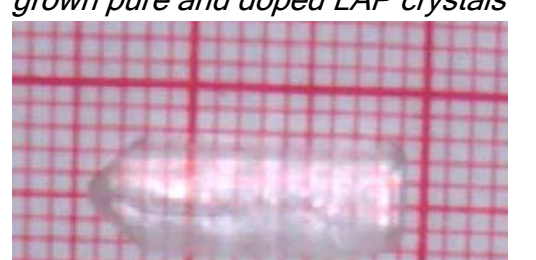

grown crystals were subjected to various characterization measurements.

Experiments

All the starting materials were of analytical reagent grade and used as purchased. LAP was prepared by mixing L-arginine with orthophosphoric acid in 1:1 mole ratio. The mixture was placed in a magnetic stirrer and stirred for about 3 hours. Long time stirring was done for the homogeneous mixing of the mixture. In the solution growth technique, selection of a solvent which is moderately soluble plays an important role. In the present study water was used as the solvent for synthesis of the compound and growth of LAP crystals. The synthesized salt of LAP was used for solubility test and crystal growth after repeated recrystallization. The recrystallized LAP was dissolved in $100 \mathrm{ml}$ of water at $40^{\circ} \mathrm{C}$. After the saturated solution of LAP was obtained, it was filtered using a filter paper after preheating. The solution was optimally covered using a plane thin plastic sheet and placed in a constant temperature bath for crystal growth. Crystals of LAP were grown from its saturated aqueous solution by the slow evaporation technique within 7 days. Transparent crystals with high optical-quality were selected from the harvested crystals for characterization studies. Doped crystals were obtained by adding the dopants to LAP solution in the required molar concentration. The dopant concentration used in this study was $0.01 \mathrm{M}$. The dopant (urea or thiourea) was mixed with the pure

"L-arginine phosphate crystal" http://www.indjst.org
Sumithraj et al. Indian J.Sci.Technol. 
Fig. 2. FTIR spectrum of LAP crystal

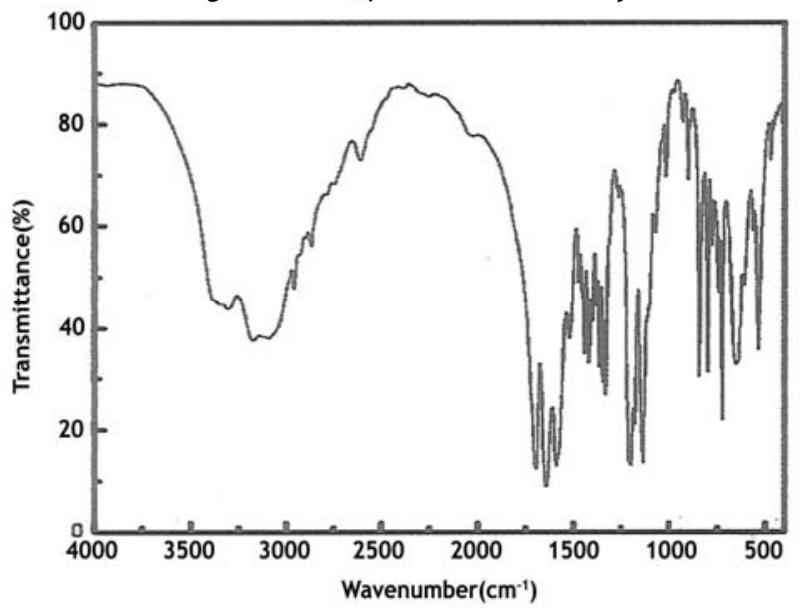

Fig. 4. FTIR spectrum of thiourea doped LAP crystal

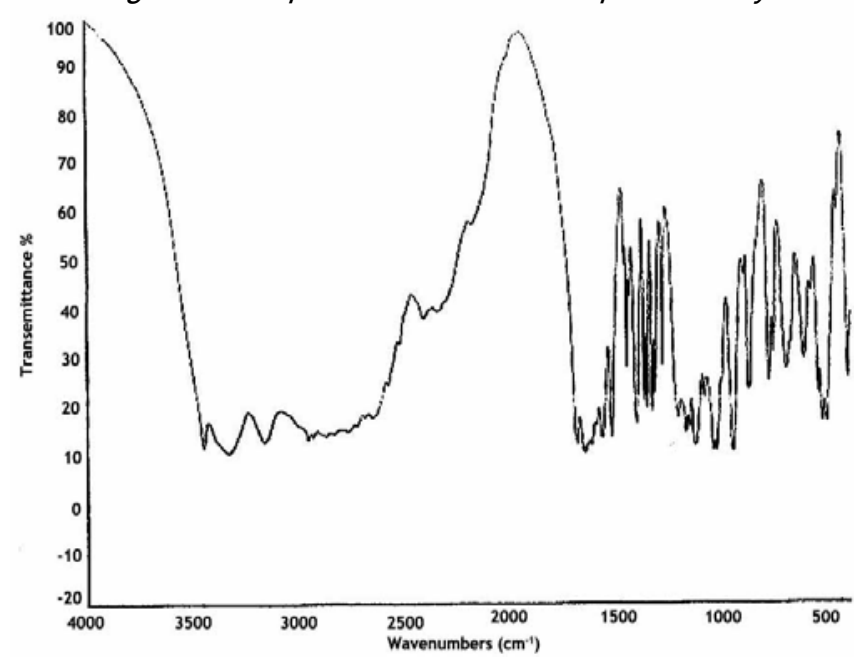

Table 1. Wavenumbers of absorption peaks and corresponding band assignment of the grown crystals

\begin{tabular}{|c|c|c|c|}
\hline \multicolumn{3}{|c|}{$\begin{array}{c}\text { Wave numbers } \\
\text { (in cm-1) of the } \\
\text { absorption peaks }\end{array}$} & \multirow{2}{*}{ Band assignments } \\
\cline { 1 - 2 } LAP & $\begin{array}{c}\text { urea } \\
\text { doped }\end{array}$ & $\begin{array}{c}\text { thiourea } \\
\text { doped }\end{array}$ & \\
\hline 3450 & 3449 & 3449 & $\mathrm{O}-\mathrm{H}$ or N - H stretching \\
\hline 3334 & 3332 & 3331 & $\begin{array}{c}\mathrm{N}-\mathrm{H} \text { stretching of } \\
\text { the aliphatic secondary } \\
\text { amine }\end{array}$ \\
\hline 3166 & 3165 & 3164 & $\begin{array}{c}\mathrm{N}-\mathrm{H} \text { stretch of the } \\
\text { primary amine }\end{array}$ \\
\hline 2959 & 2961 & 2961 & $\mathrm{C}=\mathrm{N}-\mathrm{H}$ stretching \\
\hline 1690 & 1690 & 1689 & $\mathrm{C}=\mathrm{O}$ stretching \\
\hline 1651 & 1651 & 1650 & $\mathrm{COO}$ - amine \\
\hline- & - & 1371 & $\mathrm{C}=\mathrm{S}$ stretching \\
\hline
\end{tabular}

Vol. 3 No. 3 (Mar 2010)

ISSN: 0974- 6846

Fig. 3. FTIR spectrum of urea doped LAP crystal
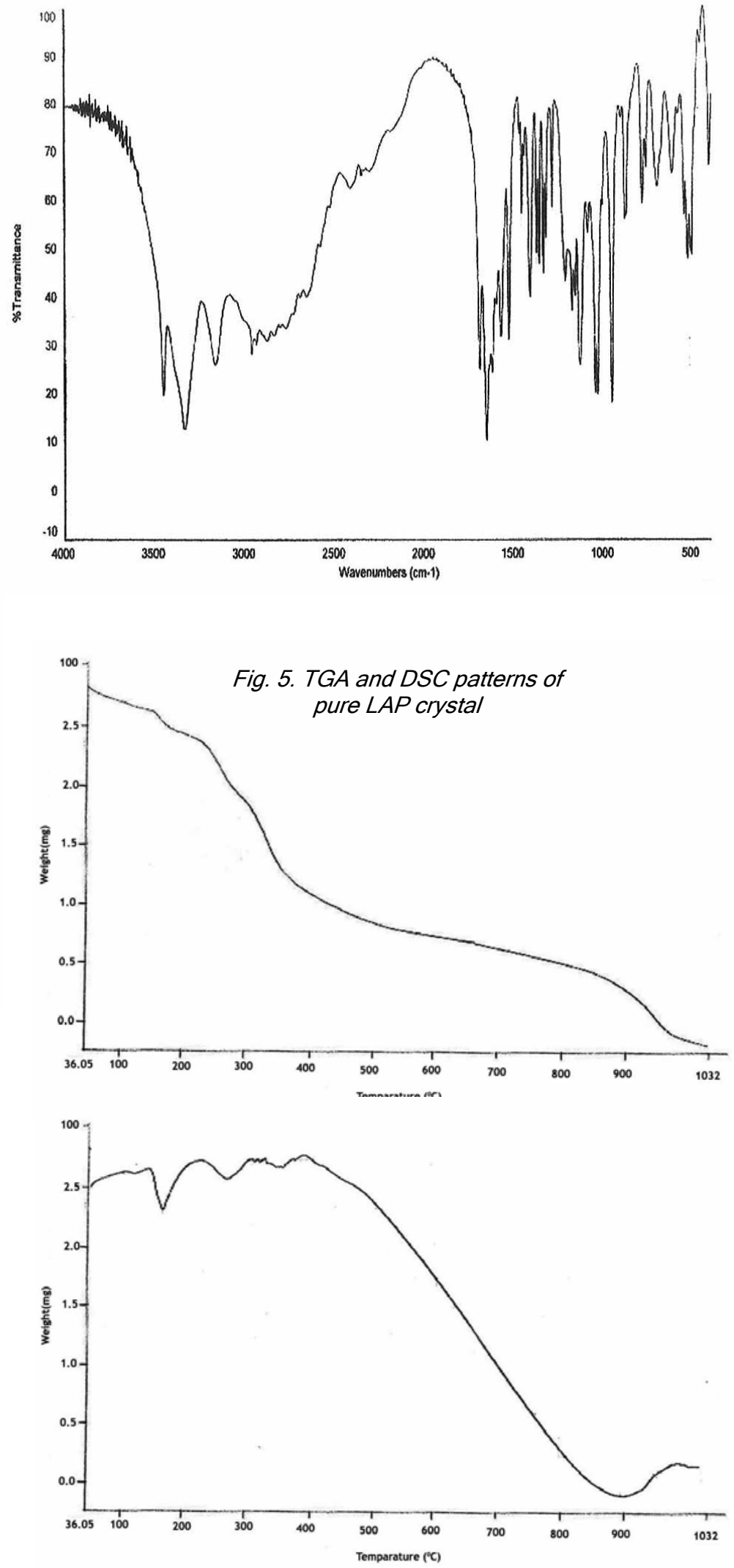

Research article

CIndian Society for Education and Environment (iSee)
"L-arginine phosphate crystal" http://www.indjst.org
Sumithraj et al. Indian J.Sci.Technol. 
solution directly.

The vibrational measurements were carried out at room temperature. Fourier transform infrared spectra were obtained from potassium bromide pellets on a Thermo Nicolet, Avatar 370 FT-IR spectrometer in the region $4000-400 \mathrm{~cm}^{-1}$. Thermo gravimetric analysis and differential scanning calorimetry analysis (TGA/DSC) were carried out in $40-800^{\circ} \mathrm{C}$ temperature range using a Perkin Elmer, Diamond TG/DTA instrument with a heating rate $10^{\circ} \mathrm{C} / \mathrm{min}$ in nitrogen atmosphere. Samples were weighed in a platinum crucible with a microprocessor-driven temperature control unit and a data station. The SHG measurement was performed using the technique of Kurtz and Perry. An Nd: YAG laser with fundamental radiation of $1064 \mathrm{~nm}$ was used as the optical source and directed onto the powder samples.

\section{Results and discussion Crystal growth}

The doped crystals were found to be more transparent and perfect than the pure crystals. The morphological perfection of the doped crystals was better than the pure crystals grown in the present study. The maximum size of
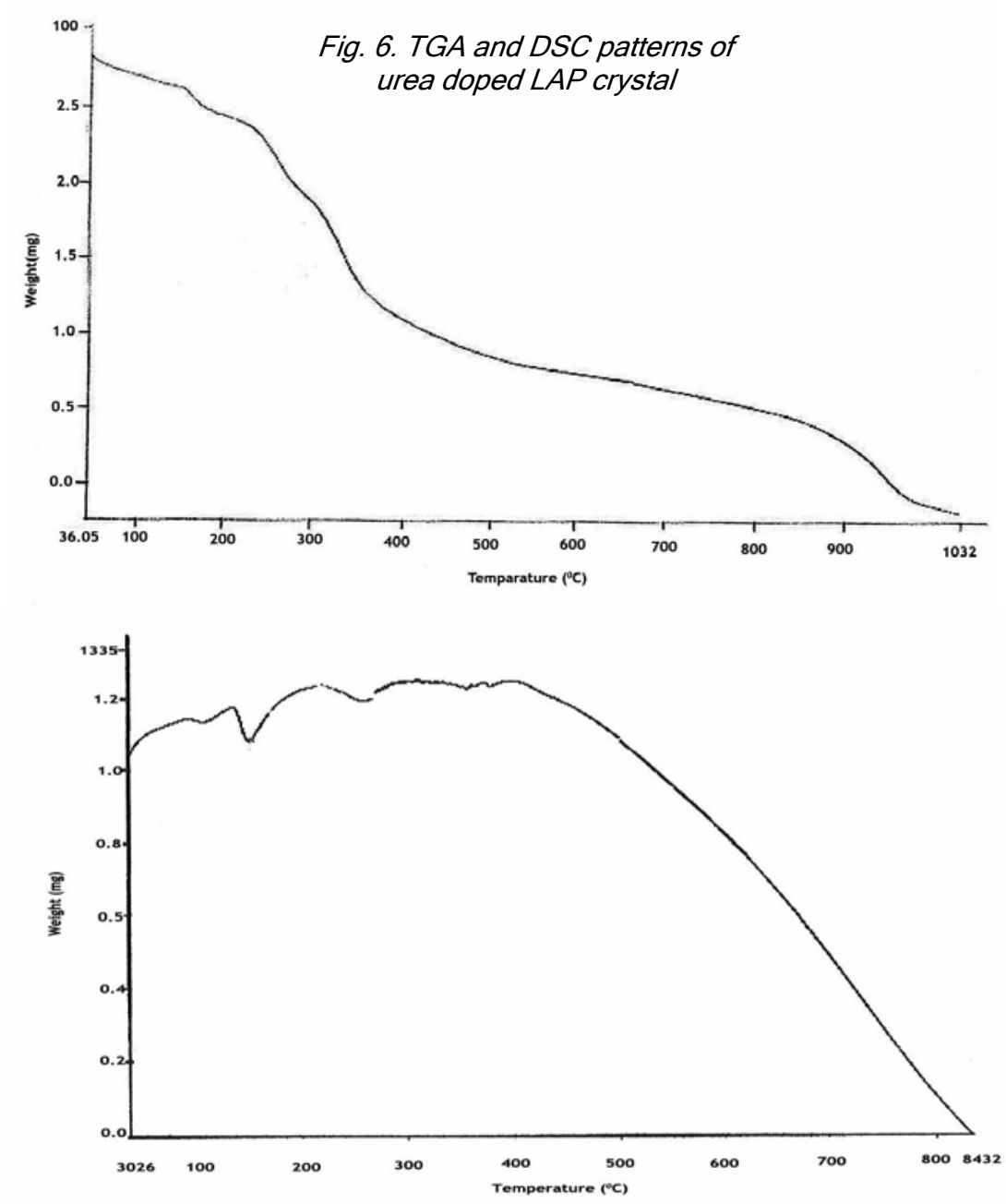

Vol. 3 No. 3 (Mar 2010)

ISSN: 0974- 6846

the pure crystals obtained in the present study was 11 $\mathrm{mm} \times 3 \mathrm{~mm} \times 3 \mathrm{~mm}$ and that of doped crystals was 14 $\mathrm{mm} \times 4 \mathrm{~mm} \times 3 \mathrm{~mm}$. The photographs of the grown crystals are shown in Fig. 1. It is observed that addition of urea or thiourea to LAP solution reduces the nucleation density and crystals grown were seen to have good morphological perfection than the pure crystal. The grown crystals were found to be non-hygroscopic and remain stable for more than 6 months, even after exposure to sunlight.

\section{FTIR studies}

The FTIR spectra recorded for the grown crystals are presented in Fig. 2-4. The band assignments are presented in Table 1. The FTIR spectra and the corresponding band assignment clearly indicate that the functional groups of pure LAP are not altered by the addition of the dopants. Moreover the presence of absorption peak at $1371 \mathrm{~cm}^{-1}$ confirms the presence of $\mathrm{C}=\mathrm{S}$ stretching in the thiourea doped LAP crystal confirming that the dopant has entered into the LAP lattice which is absent in the pure and urea doped LAP crystals.

\section{Thermal studies}

The TGA and DSC curves of LAP are shown in Fig. 5-7. From the TGA curve, one can see that the material starts decomposing above $172^{\circ} \mathrm{C}$. This is supposed to be the utilization of the thermal energy in overcoming the valance bonding between the L-Arg+ cation and the phosphate anion, which happens during the initial stage of decomposition. Similar observation has been reported for L-arginine trifluoroacetate (Sun et al., 2007). At this stage of decomposition the LAP crystals loses 2 molecules. At $274^{\circ} \mathrm{C}$, the second decomposition starts with the evolution of carbon di-oxide. At $350^{\circ} \mathrm{C}$, the third decomposition starts leaving the residue at the end of the heating process. Similar observations are noted for the doped crystals also. The decomposition of pure and doped LAP crystals are supported by the corresponding endothermic dips in the respective temperatures obtained in the DSC analysis. The expected stages of decomposition along with the $\%$ weight loss and expected loss of molecules are presented in Table 2.

\section{NLO studies}

SHG may be employed as a tool to evaluate at least qualitatively the bulk homogeneity of the crystals under investigation. To check the nonlinear optical property of the grown crystal, Kurtz and Perry

Research article

CIndian Society for Education and Environment (iSee)
"L-arginine phosphate crystal" http://www.indjst.org
Sumithraj et al. Indian J.Sci.Technol. 

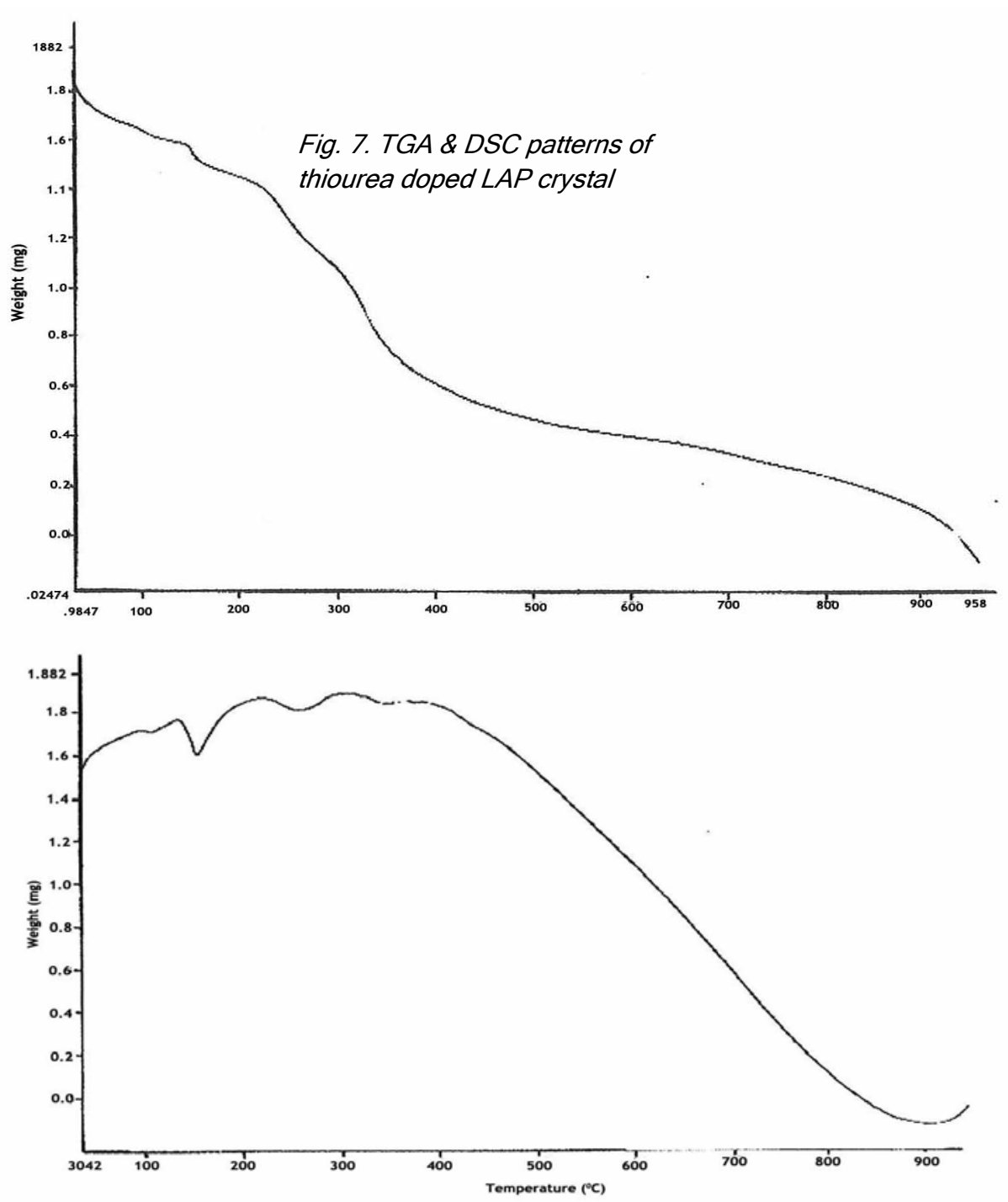

\section{References}

1. Dhanraj G, Shripathi T and Bhat HL (1991) Growth and defect characterization of L-arginine phosphate monohydrate. J. Cryst. Growth. 113, 456-464.

2. Dmitriev VG, Gurzadyan GG and Nicogosyan DN (1999) Handbook of nonlinear optical crystals, SpringerVerlag, NY.

3. Gnanasekaran $P$ and Madhavan $J$ (2008) L- arginine acetate single crystals for NLO applications. Indian J. Sci. Technol. 7, 1-2.

4. Kurtz SK and Perry TT (1968) A Powder technique for the evaluation of nonlinear optical materials. $J$. Appl. Phys. 39, 3798-3812.

5. Munirathnam and Madhavan $J$ (2009) Investigations on the structural, mechanical and photoconductive studies of pure and lanthanum doped potassium pentaborate single crystals. Indian J. Sci. Technol. 2, 44-45.

6. Nicoud JF and Twieg RJ (1987) Nonlinear optical properties of organic molecules and crystals. Chemla DS, Zyss JE (Ed.) Academic Press, London.

7. Ravi G, Srinivasan K, Anbukumar S and Ramasamy P (1994) Growth and characterization of sulphate mixed L-arginine phosphate and ammonium dihydrogen phosphate/potassium dihydrogen phosphate mixed crystals. J. Cryst. Growth. 137, 598-604.

technique was used (Kurtz \& Perry, 1968). A highintensity $\mathrm{Nd}$ : YAG laser with fundamental radiation of $1064 \mathrm{~nm}$ was used as the optical source and directed onto the powder sample of LAP. The SHG behaviour was confirmed from the output of intense green light emission $(\lambda=532 \mathrm{~nm})$ from the crystal. Intensity of the bright green emission was estimated to be 2.5 times more than that of the standard KDP crystal.

\section{Conclusion}

In this paper, the synthesis as well as single crystal growth of LAP has been reported. LAP crystals were grown by conventional low temperature solution growth method. FT-IR spectra confirm the various functional groups present in the grown crystal. The thermal studies on the grown crystals show that these crystals are thermally stable up to $172^{\circ} \mathrm{C}$. The SHG intensity of LAP is found to be 2.5 times more than that of KDP, which shows that the crystal is a potential NLO material.
8. Sun $Z H, X u D$, Wang $X Q$, Liu XJ, Yu G, Zhang GH, Zhu LY and Fan HL (2007) Growth and characterization of the nonlinear optical crystal: Larginine trifluoroacetate. Cryst. Res. Technol. 42, 812816.

9. Wong C Bosshard, Pan F and Gunter P (1996) Nonclassical donor-acceptor chromophores for second order nonlinear optics. Adv. Mater. 8, 677-680.

10.Xu D, Jiang MH and Tan ZK (1983) A new phase matchable nonlinear optical crystal L-Arginine Phosphate monohydrate. Acta. Chim. Sin. 41, 570-576. http://www.indjst.org
Sumithraj et al. Indian J.Sci.Technol. 